\title{
Chemogenetic Inhibition Reveals That Processing Relative But Not Absolute Threat Requires Basal Amygdala
}

\author{
Vincent D. Campese, ${ }^{1} \oplus^{-}$Ian T. Kim, ${ }^{2}$ Mian Hou, ${ }^{2}$ Saurav Gupta, ${ }^{1}$ Cassandra Draus, ${ }^{2}$ Botagoz Kurpas, ${ }^{3}$ Kelsey Burke, ${ }^{4}$ \\ and ${ }^{-J o s e p h ~ E . ~ L e D o u x ~}{ }^{2,5}$ \\ ${ }^{1}$ Department of Psychology, University of Evansville, Evansville Indiana 47722, ${ }^{2}$ Center for Neural Science, New York University, New York, New York \\ 10003, ${ }^{3}$ Department of Psychology, Kingsborough College, Brooklyn, New York 11235, ${ }^{4}$ Department of Psychology, Hunter College, New York, New York \\ 10065, and ${ }^{5}$ Emotional Brain Institute, Nathan Kline institute for Psychiatric Research, Orangeburg New York 10962
}

While our understanding of appetitive motivation has benefited immensely from the use of selective outcome devaluation tools, the same cannot be said about aversive motivation. Findings from appetitive conditioning studies have shown that basal amygdala is required for behaviors that are sensitive to updates in outcome value, but similar results in aversive motivation are difficult to interpret due to a lack of outcome specificity. The studies reported here sought to develop procedures to isolate sensory-specific processes in aversive learning and behavior and to assess the possible contribution of the basal amygdala. Post-training changes to outcome value produced commensurate changes to subsequently tested conditioned responding in male rodents. Specifically, increases in shock intensity (i.e., inflation) augmented, while repeated exposure to (i.e., habituation of) an aversive sound (klaxon-horn) reduced freezing to conditioned stimuli previously paired with these outcomes. This was extended to a discriminative procedure, in which following revaluation of one event, but not the other, responding was found to be dependent on outcome value signaled by each cue. Chemogenetic inactivation of basal amygdala impaired this discrimination between stimuli signaling differently valued outcomes, but did not affect the revaluation process itself. These findings demonstrate a contribution of the basal amygdala to aversive outcome-dependent motivational processes.

Key words: aversive; devaluation; discrimination; motivation; salience; value

\section{Significance Statement}

The specific content of pavlovian associative learning has been well studied in appetitive motivation, where the value of different foods can be easily manipulated. This has facilitated our understanding of the neural circuits that generate different forms of motivation (i.e., sensory specific vs general). Studies of aversive learning have not produced the same degree of understanding with regard to sensory specificity due to a lack of tools for evaluating sensory-specific processes. Here we use a variant of outcome devaluation procedures with aversive stimuli to study the role of basal amygdala in discriminating between aversive stimuli conveying different degrees of threat. These findings have implications for how we study generalized threat to identify dysregulation that can contribute to generalized anxiety.

\section{Introduction}

Studies of aversive pavlovian threat conditioning have provided an effective model for how aversive memories are formed, maintained, and expressed in relation to stress and trauma (LeDoux and Pine, 2016; Deslauriers et al., 2018). Over the past decades

\footnotetext{
Received Sept. 28, 2018; revised Aug. 26, 2019; accepted Aug. 28, 2019

Author contributions: V.D.C. and J.E.L. designed research; I.T.K., M.H., S.G., C.D., B.K., and K.B. performed research; V.D.C., I.T.K., M.H., S.G., and B.K. analyzed data; V.D.C. wrote the paper.

This research was supported by National Institutes of Health Grants R01-MH-038774 and R01-DA-044445 to

J.E.L. We thank Christopher K. Cain, Robert M. Sears, and Justin M. Moscarello for comments on an early version of this manuscript as well as for frequent discussions on related issues.

The authors declare no competing financial interests.

Correspondence should be addressed to Vincent D. Campese at vc44@evansville.edu.

https://doi.org/10.1523/JNEUROSCI.2530-18.2019

Copyright $@ 2019$ the authors
}

much has been learned about the neural circuits and molecular mechanisms that regulate aversive pavlovian conditioning and memory (Janak and Tye, 2015; for review, see Herry and Johansen, 2014; Krabbe et al., 2018). This literature presents a detailed understanding of the psychological and neurobiological nature of this simple form of learning. However, in natural settings, organisms are often faced with a multitude of threats, some more dangerous than others. In these situations, a comparison of the relative degree of threat signaled by two distinct stimuli can be useful in guiding behavior and matching defensive responding to the threat potential of particular stimuli. The discriminative processes that underlie such contrasts in aversive motivation are poorly understood.

Experimental findings indicate that pavlovian conditioning procedures result in stimulus-outcome learning (Rescorla, 1974; 
Balleine and Killcross, 2006; Díaz-Mataix et al., 2011), and that changes to the value of the outcome produce commensurate changes in conditioned behavior via neural processes in the basal amygdala (BA; Chen et al., 2011; Ghosh and Chattarji, 2015; Sengupta et al., 2018). However, much of the work demonstrating this is limited to discriminative procedures involving a shockpaired conditioned stimulus (e.g., $\mathrm{CS}^{+}$) and another cue (CS) with no aversive outcome (e.g., $\mathrm{CS}^{-}$; Chen et al., 2011; Ghosh and Chattarji, 2015; Staples-Bradley et al., 2018). Such procedures could reflect flexible outcome-selective behavior or, alternatively, less attention to irrelevant and inconsequential stimuli (Le Pelley et al., 2014; Kattner and Green, 2016).

In appetitive studies, food-motivated paradigms in which multiple stimulus-outcome associations are used in combination with devaluation or extinction procedures. These tools permit sensory-specific encoding to be more effectively assessed than in simple $\mathrm{CS}^{+}$versus $\mathrm{CS}^{-}$discrimination studies (Delamater, 1996; Rescorla, 1996; Pickens et al., 2005; Corbit et al., 2007). The former techniques have proven valuable for identifying circuits that process and update outcome value for the use of behavior dependent on pavlovian signaling (Rescorla, 1974; Schoenbaum et al., 2003a; Delamater, 2012). Furthermore, these procedures have also been used to probe the psychological and neural substrates underlying instrumental learning (Rescorla, 1991), choice (Izquierdo et al., 2004; Corbit and Balleine, 2005), and habit (Coutureau and Killcross, 2003).

Our understanding of aversive motivation could benefit from the use of similar procedures to isolate sensory-specific processes (Campese et al., 2017). Therefore, in the studies below, we have established ways to change the value of aversive unconditioned stimuli (US) following training that would permit such investigation. These revaluation procedures were tailored to two distinct aversive outcomes in separate groups of rats. In one group, following standard tone-shock pairings, unsignaled shocks were administered at a comparably higher intensity, resulting in increased (or inflated) freezing (Rescorla, 1974). Another group of rats received pairings of a tone with a loud noxious horn (i.e., a klaxon) that functioned as a US (Rescorla, 1973). Following training, subject animals were habituated to this outcome, and this reduced conditioned responding in a subsequent test. These procedures were then combined in a within-subject animals design where revaluation procedures were applied and demonstrated to be US specific. Finally, to test whether BA is important for organizing conditioned responding on the basis of relative US value, we used chemogenetic techniques involving $\kappa$-opioid designer receptors (Vardy et al., 2015; Marchant et al., 2016) to inhibit activity in this region during tests for long-term memory. These studies show that CS-elicited behavior is significantly altered by these treatments and that BA inhibition impairs the ability for relative but not absolute value to regulate the degree of conditioned responding to each CS.

\section{Materials and Methods}

\section{Subject animals}

One hundred twenty-four male Sprague Dawley rats, purchased from Hilltop Lab Animals were the subjects of the studies reported below. All rats weighed $\sim 300 \mathrm{~g}$ at the start of the experimentation. Subject animals were housed in standard Plexiglas cages with free food and water available on paper bedding in a rodent colony running a $12 \mathrm{~h}$ light/dark schedule. Animals used at the University of Evansville were bred from founders purchased from Hilltop Lab Animals, and housing conditions were identical to those at New York University. Animal care and housing was in accordance with the Institutional Animal Care and Usage Committee (IACUC) policies and met the current standards of the Associa- tion for Assessment and Accreditation of Laboratory Animal Care International. The New York University Animal Welfare Committee as well as the University of Evansville IACUC approved all of the experimental procedures reported herein.

\section{Apparatus}

Behavioral sessions took place in standard conditioning chambers (model H10-11R-TC, Coulbourn Instruments), which are referred to as context A below. For studies at the University of Evansville, chambers were manufactured by Lafayette Instrument (model 80003). Context A had stainless steel floors that delivered the $1 \mathrm{~s}$ footshock US. Two different intensities were used in the studies reported below: $0.5 \mathrm{~mA}$ for training and $1.0 \mathrm{~mA}$ for revaluation. Additionally, each chamber in context $\mathrm{A}$ was equipped with a horn for delivering the klaxon US $(114 \mathrm{~dB}, 5 \mathrm{~s}$ duration; model 330, Wolo). Context $B$ was another set of identical Coulbourn Instruments or Lafayette Instrument chambers used for test sessions. To produce perceptually distinct contexts, flat plastic floors were inserted in addition to black and white striped walls as well as peppermint scent (Dr. Bronner's Magic Soaps) to the waste trays. Chambers in both contexts were equipped with a $5 \Omega$ speaker and a programmable Coulbourn Instruments audio generator (model A12-33) to deliver the $5 \mathrm{kHz}$ tone and white noise stimuli (in decibels) as well as a video camera to record footage from the behavioral sessions for later analyses. Auditory stimuli at the University of Evansville were produced by Med Associates ANL-926 modules. All other accessories were as described above.

\section{Procedure}

Context habituation. Subject animals were exposed to contexts A and B for $15 \mathrm{~min}$ each on day 1 of the study. During exposure, there were $30 \mathrm{~min}$ between each session and context order was counterbalanced. While house lights were on, no other stimuli were presented, and subject animals spent the intervening time in their home cages in the vivarium.

Pavlovian conditioning. On the day after context exposure, subject animals received pavlovian conditioning in context A. Three trials occurred where a $30 \mathrm{~s}$ auditory stimulus, tone or white noise, coterminated with either a footshock or a klaxon horn. Trials were separated by a 3 min intertrial interval (ITI) with the total session duration at $15 \mathrm{~min}$. Following this session, subject animals were returned to the vivarium and remained there for the rest of the day. If subject animals required a second training session for a subsequent within-subject animals comparison, this was also conducted in context A, $24 \mathrm{~h}$ after their first training session (see Fig. $3 E$ for experimental designs table). The session parameters were the same as those on day 1 with the exception of the CS and US identity. For example, subject animals that had tone-shock pairings on day 1 received noise-klaxon pairings on day 2 . In this case, both session order and stimulus-outcome assignments were counterbalanced.

Revaluation. Beginning on the day following the end of pavlovian conditioning, subject animals received US revaluation treatments in context A in the absence of the CS. Shock inflation was accomplished by presenting six unsignaled footshocks with the same temporal parameters and spacing as was used during acquisition. While controls received US of the same intensity during this phase as they did during acquisition (i.e., $0.5 \mathrm{~mA}$ ), "inflated" subject animals received more intense $1.0 \mathrm{~mA}$ footshocks. This occurred over a single 24 min session. Klaxon habituation occurred over the $3 \mathrm{~d}$ following the end of pavlovian training in context A. In these sessions, subject animals received 24 unsignaled $5 \mathrm{~s}$ klaxon presentations separated by $15 \mathrm{~s}$ after a 3 min baseline period, while controls were simply placed in the context and received no stimuli. There was a 1 min rest period after the last klaxon presentation. For discrimination studies, controls also received context exposure during the revaluation phase without any other stimulation. These sessions were $12 \mathrm{~min}$ in total duration.

Testing. The test session occurred in context $\mathrm{B}$ the day after revaluation had ended and included three trials with a 30 s CS (e.g., tone or noise) without any US presentation. Trials were separated by a 3 min ITI, and sessions were a total of $15 \mathrm{~min}$ in duration. For subject animals tested with two stimuli for the discrimination task, the session duration was doubled and the order of presentations was counterbalanced so that half of the subject animals received three tone trials followed by three noise 
trials, and vice versa. The differences between the training (context A) and test location (context B) have been shown to produce perceptually distinct and discriminable contexts in previous work (Campese and Delamater, 2013, 2014).

Delivery, activation, and verification of $\kappa$ opioid receptor designer. For the delivery of $\mathrm{G}_{\mathrm{i}}$-coupled $\kappa$ opioid receptor designer (KORD) receptors exclusively activated by designer drugs (Vardy et al., 2015; Marchant et al., 2016; University of North Carolina Vector Core, Chapel Hill, NC) to the BA, subject animals were anesthetized with an intraperitoneal injection of a ketamine $(100 \mathrm{~kg} / \mathrm{mg}) /$ xylazine $(10 \mathrm{mg} / \mathrm{kg})$ mixture (VedCo). Once sedated, subject animals were prepared and placed into a stereotaxic apparatus (David Kopf Instruments). BA was targeted at the following coordinates relative to bregma $(3.1 \mathrm{~mm}$ posterior, $5.2 \mathrm{~mm}$ lateral, and $9 \mathrm{~mm}$ ventral). Through a $1 \mu \mathrm{l}$ Neuros syringe (model 7001, Hamilton), $0.7 \mu \mathrm{l}$ of adeno-associated virus 9 CaMKII containing instructions for KORD was injected bilaterally into BA over $5 \mathrm{~min}$ and allowed to spread for an additional $5 \mathrm{~min}$ before removing the needle over an additional 1 min period. subject animals were sutured and given analgesia (ketaprofen, $5 \mathrm{mg} / \mathrm{kg}$, s.c.) postoperatively over $3 \mathrm{~d}$ in their home cages with free food and water available.

Salvinorin-B (Sal-B; Apple Pharms) was dissolved in dimethylsulfoxide ( $7 \%$ of total volume; Sigma-Aldrich) and added to warm $50^{\circ} \mathrm{C}$ polyethylene glycol (Sigma-Aldrich) and deionized water (1:1) to produce a $15 \mathrm{mg} / \mathrm{ml}$ solution. subject animals received $0.1 \%$ body weight intraperitoneal treatment with Sal-B or vehicle 15 min before testing.

Following the study, subject animals were given an overdose of ketamine/xylazine $(1 \mathrm{ml})$ and perfused transcardially with physiological PBS, then fixed with $4 \%$ paraformaldehyde (PFA) in $0.2 \mathrm{M}$ phosphate buffer. Following postfixing in $4 \%$ PFA, brains were cut into $50 \mu \mathrm{m}$ coronal sections on a Vibratome (Leica Biosystems), stored in PBS with $0.05 \%$ sodium azide, and kept at $40^{\circ} \mathrm{C}$ until processing. The expression of KORDs was visualized using a Life Technologies rabbit anti-GFP antibody (1:2000; \#A11122; Thermo Fisher Scientific), biotinylated goat anti-rabbit IgG (1:200; Vector Laboratories), avidin-biotin horseradish complex (Elite ABC Kit; Vector Laboratories), and very intense purple (VIP Kit, Vector Laboratories). Sections were mounted, coverslipped, and viewed using a high-resolution digital camera microscope system (catalog \#VS120, Olympus).

Cellular responses to Sal-B. A subset of subject animals from the current studies was used to verify the efficacy and selectivity of Sal-B inhibition in KORD-expressing neurons. Brain slices were prepared from subject animals expressing KORD (five slices from four rats) and experimentally naive and nonoperated controls matched for age and weight (seven slices in three rats) as in the study by Johnson et al. (2008) and maintained on an interface chamber at $31^{\circ} \mathrm{C}$. Glass recording electrodes (filled with aCSF, $5 \mathrm{M} \Omega$ resistance) were guided to dorsal striatum neurons receiving projections from basal amygdala. Bipolar stainless steel stimulating electrodes $(75 \mathrm{k} \Omega)$ were positioned to target projection fibers. Orthodromic synaptic potentials were evoked via an isolated current generator $(100 \mu \mathrm{s}$ pulses of 0.3-0.7 mA; Digitimer). Evoked field potentials were recorded with an Axoclamp 2B amplifier and Axon WCP software (Molecular Devices). Data were analyzed off-line using WCP PeakFit (Molecular Devices). Field EPSP (fEPSP) was measured as a change in evoked field potential amplitude. Baseline responses were monitored at $0.05 \mathrm{~Hz}$ for 30 min with a stimulus intensity of $40-50 \%$ of maximum fEPSP before Sal-B (100 nM) application, and this intensity was maintained for the rest of recording.

Experimental design and statistical analysis. In simple revaluation studies (i.e., those involving a single CS-US associative relationship), purely between-groups designs were used and independent $t$ tests were implemented to evaluate the effect of revaluation relative to nontreated controls. The same strategy was applied to analysis of freezing during the baseline (or pre-CS) interval, which was defined as $30 \mathrm{~s}$ before each CS. Studies involving discrimination between multiple CS-US associations following revaluation were accomplished using within-subject animals procedures. subject animals were trained independently with each CS-US treatment and had only one outcome revalued (i.e., shock, in studies using viral inhibition). Data from studies including these arrangements were analyzed using repeated-measures ANOVA (rmANOVA)
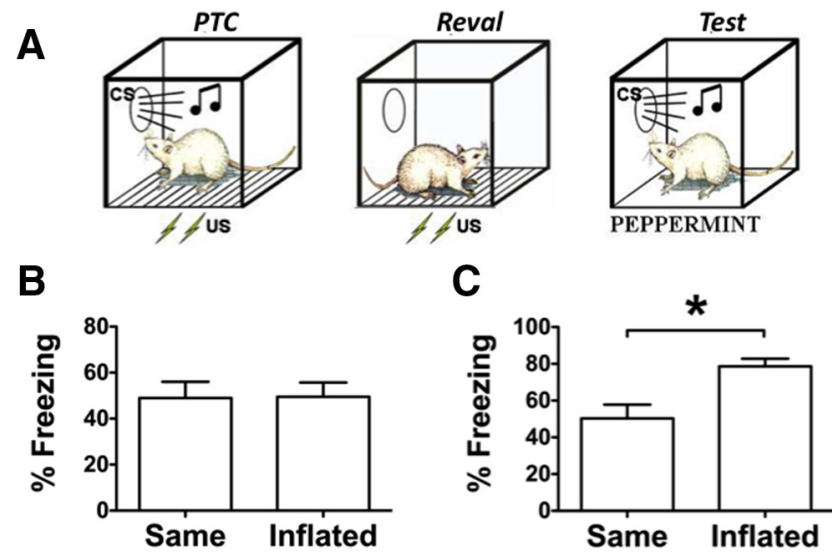

Figure 1. $\quad \boldsymbol{A}$, The experimental design for simple and absolute inflation. $\boldsymbol{B}, \boldsymbol{C}$, Freezing is presented in terms of the percentage of time for pavlovian threat conditioning (PTC; $\boldsymbol{B})$ and the test session (C). ${ }^{*}$ Significance at $\alpha=0.05$.

approaches with mixed models used to test whether viral inhibition changed behavioral patterns across the two CSs. Pre-CS data from these studies were analyzed using the same approach.

\section{Results}

Data from the pavlovian conditioning (Figs. $1 B, 2 B$ ) and test phases (Figs. $1 C, 2 C$ ) are presented in terms of the percentage of time freezing to the CS for shock-inflation and klaxon-habituation groups, respectively. For these groups, training and test data were analyzed with independent-samples $t$ tests. In both cases these analyses found that control and experimental subject animals froze comparably to the CS during pavlovian conditioning (shock-inflation group: $t_{(14)}=0.06, p=0.95$; klaxon-habituation: $t_{(13)}=0.67, p=0.52$; Figs. $1 B, 2 B$ ). Preliminary analyses found that there were no differences in pre-CS responding for control and revalued subject animals in the shock-inflation subset $\left[t_{(14)}=0.61, p=0.55\right.$; mean values: control $=50.42(\mathrm{SEM}=$ $7.9)$, inflated $=42.22(\mathrm{SEM}=10.8)]$ and the klaxon-habituation subset $\left[t_{(13)}=0.36, p=0.73\right.$; mean: control $=30.16(\mathrm{SEM}=$ $2.7)$; habituated $=25.56(\mathrm{SEM}=2.9)]$. Inspection of the CSbased test data suggests that revaluation treatments successfully influenced subsequent CS-elicited freezing. The analysis confirmed this impression: shock-inflation resulted in significantly more CS-elicited freezing compared with control treatment with the same intensity US as used during pavlovian training $\left(t_{(14)}=\right.$ $3.31, p=0.005$; Fig. 1C). Furthermore, analysis of test data for the klaxon-habituation group showed that these subject animals exhibited significantly less CS-elicited freezing than controls exposed only to the context $\left(t_{(13)}=3.31, p=0.006\right)$. Data from a follow-up test (Fig. 2D) confirmed that the reduction in CSelicited freezing for klaxon-habituated subject animals was not due to hearing damage during revaluation as both the klaxonhabituated group and the controls showed comparable freezing to a different CS (i.e., white noise) after it was paired with a 0.7 $\mathrm{mA}$ footshock $\left(t_{(13)}=0.73, p=0.48\right)$.

For discrimination subject animals, mean freezing for each group to the different CSs from the test session can be seen in Figure $3 A$. Preliminary analysis, with a split-plot rmANOVA with stimulus (CS-shock vs CS-klaxon-baseline only) as the withinsubject animals factor and group (context vs habituation vs inflation) as the between-subject animals factor, found that pre-CS responding was low, but was comparable for each group, indicating that neither inflation nor habituation affected baseline responding relative to the control group [stimulus: $F_{(1,21)}=0.13$, 

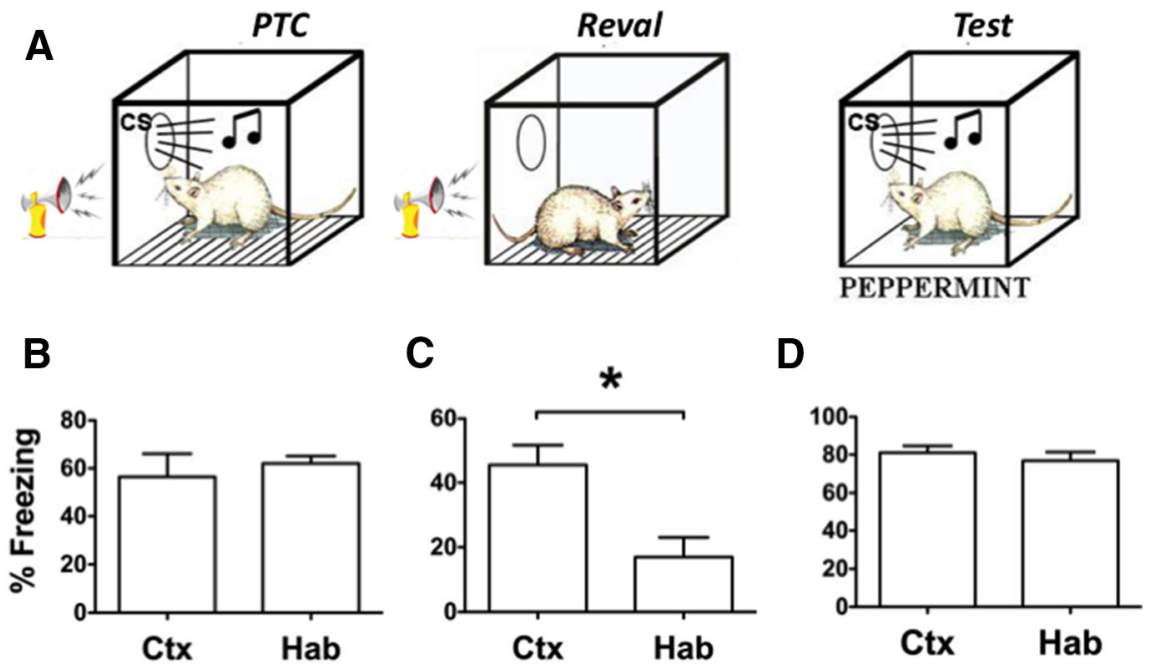

Figure 2. $\quad \boldsymbol{A}$, The experimental design for habituation with the klaxon outcome. $\boldsymbol{B}, \boldsymbol{C}$, Freezing is again presented in terms of the percentage of time for the pavlovian conditioning phase $(\boldsymbol{B})$ and the subsequent test session (C). $\boldsymbol{D}$, Freezing from a follow-up test where conditioned freezing to a shock-paired white noise was measured to determine whether klaxon habituation produced a hearing deficit. *Significance at the $0.05 \alpha$ level. Hab, Habituation; PTC, pavlovian threat conditioning; Ctx, context-exposed control.
A

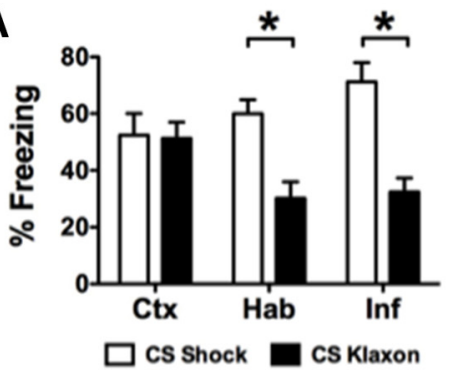

B
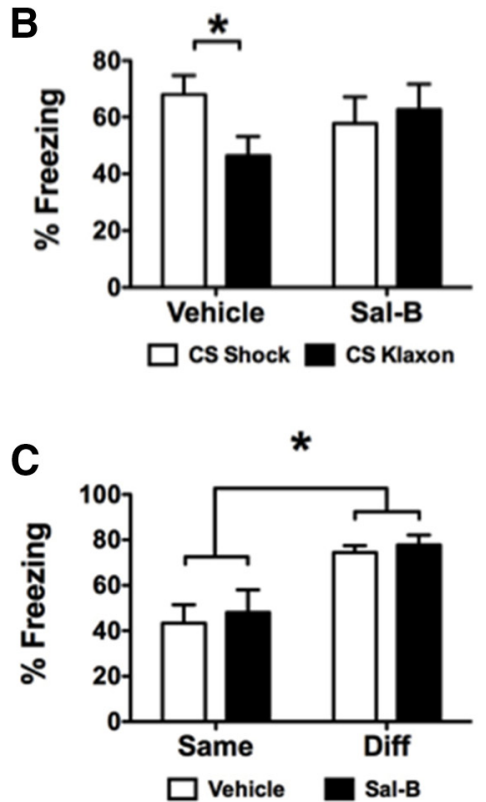

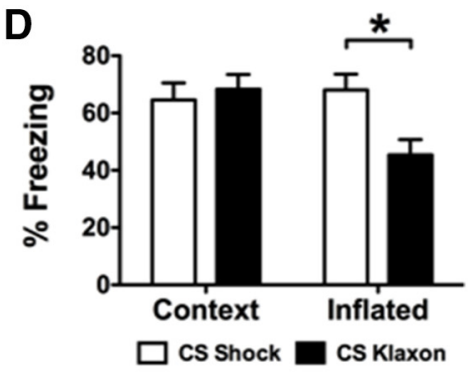

E

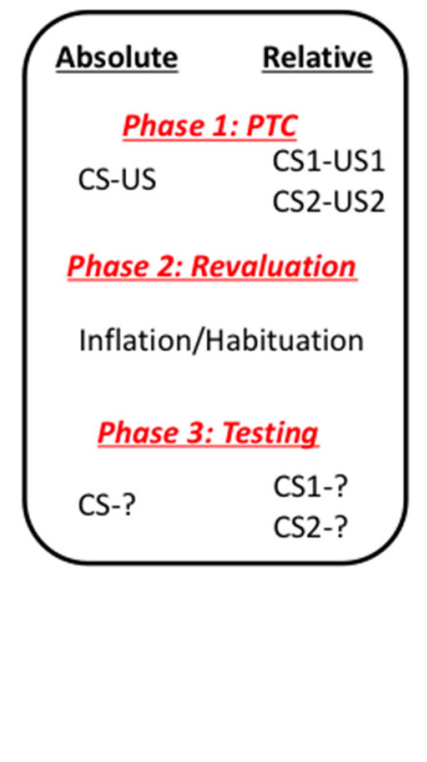

Figure 3. $\boldsymbol{A}$, Test data from the within-subject animal discrimination experiment. These data are displayed in terms of the percentage of time freezing to each CS as a function of the signaled outcome for the context-exposed controls (Ctx) as well as the inflated (Inf) and habituated (Hab) experimental groups. B. Test data from BA KORD-expressing subject animals that had undergone inflation and were treated with either Sal-B or the vehicle before the discrimination test session. $C$, Test data for BA KORDexpressing subject animals that had undergone inflation in a simple nondiscrimination design and were treated with either Sal-B or vehicle before the single-CS test session. $\boldsymbol{D}$, Test data from controls treated with Sal-B but without KORD expression. $\boldsymbol{E}$, The progression of experimental phases for studying changes to absolute and relative threat. * Significant effects at $\alpha=0.05$. $p=0.91 ;$ mean: context $=0.9(\mathrm{SEM}=$ $0.8)$; habituated $=0.4(\mathrm{SEM}=0.6)$; inflated $=6.9(\mathrm{SEM}=4.0)]$. As this analysis found no evidence of a stimulus $\times$ group interaction $\left(F_{(1,14)}=0.33, p=0.72\right)$, subsequent analyses focused only on CS responding. While freezing was comparable for the two CSs for controls, revaluation significantly changed the way subject animals responded to these stimuli. The CSresponding data were similarly analyzed, and they revealed a significant main effect for stimulus $\left(F_{(1,21)}=28.61, p<0.001\right)$ and a significant interaction between stimulus and group $\left(F_{(2,21)}=6.74, p=0.005\right)$ with no main effect for the between-subject animals factor group $\left(F_{(2,21)}=0.72, p=0.50\right)$. Bonferroni-corrected comparisons identified the following as sources of the group $X$ stimulus interaction. Freezing to the two CSs was similar in controls [CS-shock: mean $=15.75(95 \%$ CI, 11.70-19.80); CS-klaxon: mean $=15.38$ (95\% CI, 12.03 $18.72)]$. Both revaluation treatments successfully changed the distribution of conditioned freezing across these stimuli. For the klaxon-habituation group, freezing was significantly lower in response to CSklaxon [mean $=9.0(95 \%$ CI, 5.6612.34)] than to CS-shock [mean $=17.88$ (95\% CI, 13.83-21.93)]. The same was true for the shock-inflation group (CSshock: mean $=21.13$ (95\% CI, (17.0825.18); CS-klaxon: mean $=9.63(95 \% \mathrm{CI}$, 6.28-12.97)]. Thus, klaxon-habituation resulted in less freezing to CS-klaxon without significantly changing CS-shock freezing levels relative to controls [group habituation: CS-shock context group: mean = 17.88 (95\% CI, 11.7-19.8)]. However, shock-inflation had a similarly depressive impact on freezing to CS-klaxon [group inflation: CS-klaxon context group, mean $=9.63(95 \%$ CI, 12.0-18.7)], but also significantly increased freezing to CSshock relative to the control group [ group inflation: CS-shock context group, mean = 21.13 (95\% CI, 11.7-19.8)].

The test data for subject animals expressing KORD in BA following shockinflation are presented in Figure $3 B$ in terms of the percentage of time freezing to the stimuli as a function of the signaled outcome. Preliminary analyses found that pre-CS responding did not differ on the basis of stimulus or treatment [stimulus: $F_{(1,14)}=2.39, p=0.15$; interaction: $F_{(1,14)}$ $=0.002, p=0.97$; vehicle-treated subject animals: CS-shock, mean $=40.97$ $(\mathrm{SEM}=12.7)$; CS-klaxon, mean $=47.92$ $($ SEM $=13.2)$; Sal-B-treated subject animals: CS-shock, mean $=48.61($ SEM $=$ 12.5); CS-klaxon, mean $=57.92(\mathrm{SEM}=$ 
A

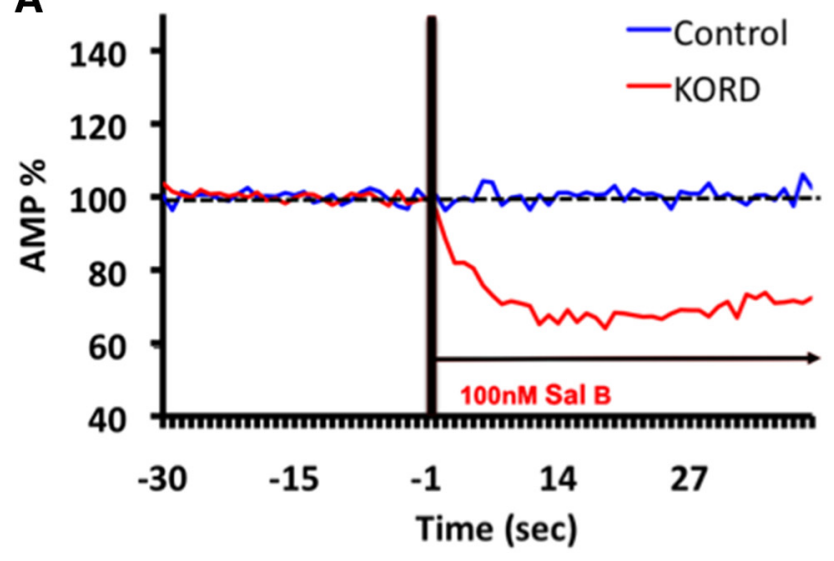

B

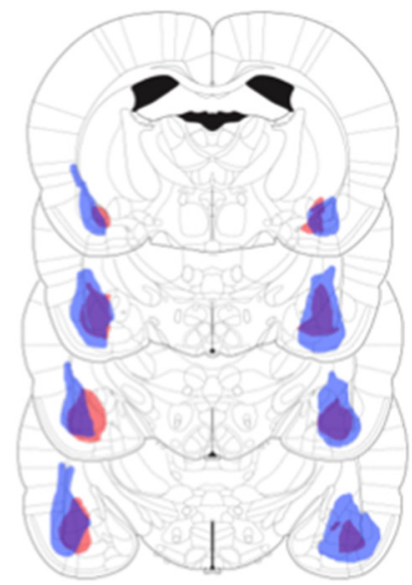

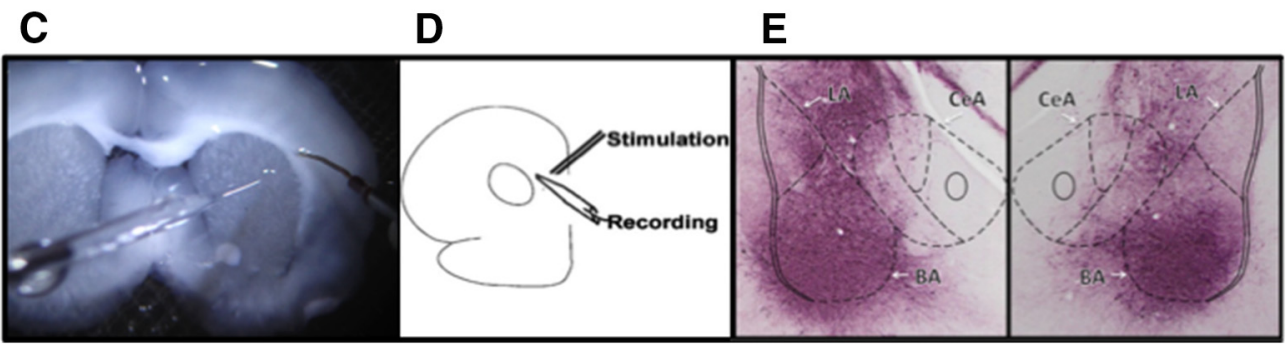

Figure 4. A, Responses of cells during a baseline period and following Sal-B treatment. $\boldsymbol{B}$, The minimum (red) and maximum (blue) extent of viral expression for subject animals treated with KORDs at the 1.56, 2.04, 2.64, and $3.24 \mathrm{~mm}$ posterior to bregma (images adapted from Paxinos and Watson, 2013). C, An image of a coronal section being tested for responses. $D$, The location of stimulating and recording electrodes can be seen in this image as well as the in schematic. $E$, A representative coronal section expressing KORD in BA using very intense purple (VIP).

10.5)]. The CS-freezing data were analyzed using a $2 \times 2$ splitplot rmANOVA with stimulus (CS-shock vs CS-klaxon) as the within-subject animals factor and treatment (Sal-B vs vehicle) as the between-subject animals factor. This ANOVA found a significant interaction between stimulus and treatment $\left(F_{(1,14)}=4.85, p=\right.$ $0.045)$. Neither the main effect of treatment $\left(F_{(1,14)}=0.09, p=0.77\right)$ nor of stimulus alone were significant $\left(F_{(1,14)}=1.61, p=0.23\right)$. Bonferroni-corrected comparisons found that while subject animals treated with the vehicle before the test showed higher freezing to CS-shock than to CS-klaxon [CS-shock: mean $=20.38$ (95\% CI, 15.13-25.62); CS-klaxon: mean $=13.88$ (95\% CI, 8.7219.03)], Sal-B-treated subject animals did not show any difference in freezing between the two cues [CS-shock: mean $=17.13$ (95\% CI, 11.89-22.37); CS-klaxon: mean $=18.88$ (95\% CI, 13.72-24.03)]. No other comparisons were significant.

To confirm that this reflects impaired discriminative processes specifically, additional BA KORD-expressing subject animals were given shock-inflation treatment without having experienced any other stimuli and were tested for CS-elicited freezing (i.e., using the single-CS and single-US design). Preliminary analyses found that there were no differences in baseline responding for these subject animals $\left[t_{(14)}=0.39, p=0.70\right.$; vehicle: mean $=58.89($ SEM $=7.1)$; Sal-B: mean $=62.92$ $(\mathrm{SEM}=7.6)]$. KORD inhibition of BA had no influence on the absolute levels of freezing when only one outcome value was tracked (Fig. $3 C$ ) compared with the discriminative (or relative) condition (Fig. 3B); both vehicle- and Sal-B-treated shockinflated groups showed more CS-elicited freezing compared with controls receiving the same shock intensity (inflation: $F_{(1,12)}=$ 19.52, $p=0.001$; treatment: $F_{(1,12)}=0.44, p=0.52$; treatment $\times$ inflation: $\left.F_{(1,12)}=0.004, p=0.95\right)$. To account for potential peripheral effects of Sal-B in the discrimination study, a group of subject animals not expressing KORD was treated with Sal-B before the test session. These subject animals received identical behavioral and pharmacological treatments as in subject animals tested for the effect of BA inactivation on the two-outcome discrimination task. The distinction being that these subject animals received no surgical treatment and were administered Sal-B before testing memory for tone and noise. Thus, if Sal-B caused peripheral effects that interfered with the discrimination, this should also be evident in these subject animals. Preliminary analyses showed no significant effects of treatment on baseline behavior [inflation: $F_{(1,18)}=0.01, p=0.93$; stimulus: $F_{(1,18)}=2.25, p=$ 1.51 ; treatment $\times$ inflation: $F_{(1,18)}=0.01, p=0.92$; contextexposed subject animals: CS-shock, mean $=30.4(\mathrm{SEM}=11.8)$; CS-klaxon, mean $=26.89($ SEM $=10.9)$; inflated subject animals: CS-Shock, mean = $31.3($ SEM = 8.9); CS-klaxon, mean = $28.2($ SEM $=8.5)]$. These results (Fig. $3 D)$ replicate the basic discrimination effect with inflation treatment (inflation: $F_{(1,18)}=$ $1.83, p=0.19$; stimulus: $F_{(1,18)}=10.65, p=0.004$; treatment $\times$ inflation: $\left.F_{(1,18)}=20.87, p=0.0002\right)$ and suggest that Sal-B alone was not responsible for impaired discrimination in KORDexpressing subject animals. Follow-up Bonferroni-corrected comparisons show that while inflated subject animals showed more freezing in response to CS-shock than CS-klaxon [CSshock: mean $=68.0(95 \%$ CI, 55.90-80.11) CS-klaxon: mean $=$ 45.33 (95\% CI, 34.35-56.32)], subject animals exposed to the context during revaluation froze comparably to the two cues [CS-shock: mean $=64.56$ (95\% CI, 52.45-76.66); CS-klaxon: mean $=68.33(95 \% \mathrm{CI}, 57.35-79.32)]$. Finally, responses of cells projecting from BA to dorsal striatum were measured in a slice preparation to compare the effects of Sal-B on activity in KORD-expressing and naive control rats. These data are presented in Figure $4 A$ and show that responses during a baseline period were comparable for both groups $\left(t_{(29)}=0.31, p=0.76\right)$, but that Sal-B significantly reduced cellular responses in KORD- 
expressing animals but not in controls $\left(t_{(39)}=128.15, p<0.001\right)$. Together, these data suggest that the discrimination between CSshock and CS-klaxon based on changes to relative value requires processing in BA but that absolute value changes do not (Fig. 3E, design comparisons). The replication of the relative revaluation effect in Sal-B-treated subject animals without KORD suggests that the deficit in KORD-expressing subject animals was due to the action on cellular responses seen in the slice study and not to peripheral effects of the drug.

\section{Discussion}

The studies reported here replicate and extend the findings that following pavlovian training footshock inflation or aversive noise (klaxon) exposure have the capacity to alter the vigor of the conditioned response (Rescorla, 1973, 1974). In both cases, responding to the CS was significantly different from controls that had not undergone adjustments. Specifically, shock inflation caused increased conditioned freezing behavior, while klaxon habituation resulted in reduced conditioned freezing behavior relative to controls. When these treatments were administered in subject animals that had been previously trained in a discriminative paradigm with both outcomes, revaluation biased defensive responding to the CS signaling the higher threat value outcome relative to the other. Interestingly, shock-inflation appeared to have a behavioral contrast-like effect, where increases in outcome magnitude that elevate one response can simultaneously cause reductions in behavior for nonadjusted outcomes in a choice task (Flaherty and Largen, 1975; Flaherty et al., 1995). There are procedural as well as qualitative differences between inflation and habituation that can potentially account for the difference. For example, habituation should reduce the threat associated with klaxon anticipation, while inflation should have the opposite psychological impact of conveying more threat. Additionally, klaxon habituation took place over $3 \mathrm{~d}$, while shock-inflation required a single session. While we did not systematically control these factors, they may have contributed to this type of adjustment to the alternate CS-US association in this discrimination task.

Using KORDs, we found that discriminating between differentially valued associative cues, but not outcome revaluation in general, is dependent upon BA. Specifically, subject animals expressing KORDs in BA performed comparably to saline-treated controls following revaluation in the single-US inflation study. On the other hand, subject animals trained with multiple outcomes had difficulty discriminating the relative values of the signaled outcomes when BA was inhibited with Sal-B treatment via KORDs. The effect was replicated and intact in vehicle-treated KORD-expressing subject animals. It should also be noted that studies in appetitive learning have suggested that learning about multiple outcomes promotes the encoding of sensory features that may not be involved in single-US designs (Balleine et al., 2003). Chemogenetic inhibition of BA in our studies may have impaired discrimination between sensory features of the outcomes in the discrimination task but not the single-US task due to sensory encoding being more likely to occur in the former task but not the latter.

While these studies did not include a nonoperated Sal-Btreated group, we are confident that KORDs had the desired effect on cellular activity and that this was selective to KORDexpressing subject animals. For example, because Sal-B treatment did not impact the inflation phenomenon with a single CS, this suggests that the BA effects obtained were due to chemogenetic inhibition and not to peripheral effects of Sal-B. If peripheral effects could accrue and impact conditioned freezing, it should apply here, but this was not observed. Furthermore, the dependence of these effects of cellular inhibition in BA was also supported with cell-response data in a slice preparation. These findings demonstrate that Sal-B effectively reduces responses in KORD-expressing cells but not in control cells. Together, these factors add confidence to our interpretation that the above results are in agreement with the following previous findings that: (1) amygdala is not required for responding following revaluation with a single CS-US associative relationship (Rabinak et al., 2009); and (2) that discriminative processes based on changes to outcome value are highly dependent on this region (Schoenbaum et al., 2003b; Ghosh and Chattarji, 2015). To our knowledge, this has not been previously demonstrated with multiple aversive outcomes in a within-subject animal design.

A limitation of this study relative to those in appetitive motivation is the lack of symmetry regarding the use of both klaxon and shock revaluation treatments when analyzing BA-based value processing. The decision to focus on inflation for this purpose was made due to a procedural time difference with shockinflation requiring only a single session and klaxon-habituation requiring $3 \mathrm{~d}$. Despite this, clear behavioral differences were seen following klaxon habituation, though whether value processing following habitation depends on BA cannot be determined from these experiments.

As we have noted, while we interpreted our findings in terms of value coding, other arguments can be made that also account for these findings relating to discrimination between the sensory features of signaled outcomes. Studies of appetitive motivation have long used devaluation treatments with great success to address such issues, and there has been no analog for them in studies of aversive motivation. The procedures outlined here can be a pivotal starting point for studying the underlying psychology of threat processing in a more ecologically valid way, as organisms deal with multiple possible sources of threat concurrently in reality. These techniques can also be used to produce a better understanding of the motivational underpinnings of instrumental avoidance behavior. In addition, further investigations of aversive US devaluation can help to determine whether results such as those presented in this article depend on value or sensory specificity. While this area has been particularly impaired by the lack of adequate behavioral tools to study the underlying psychology of aversive motivation (LeDoux and Pine, 2016; LeDoux et al., 2017; Cain, 2019), we hope this work encourages future reconciliation of differences between the appetitive and aversive learning domains.

\section{References}

Balleine BW, Killcross S (2006) Parallel incentive processing: an integrated view of amygdala function. Trends Neurosci 29:272-279.

Balleine BW, Killcross AS, Dickinson A (2003) The effect of lesions of the basolateral amygdala on instrumental conditioning. J Neurosci 23:666675.

Cain CK (2019) Avoidance problems reconsidered. Curr Opin Behav Sci 26:9-17.

Campese V, Delamater AR (2013) ABA and ABC renewal of conditioned magazine approach are not impaired by dorsal hippocampus inactivation or lesions. Behav Brain Res 248:62-73.

Campese VD, Delamater AR (2014) Dorsal hippocampus inactivation impairs spontaneous recovery of Pavlovian magazine approach responding in rats. Behav Brain Res 269:37-43.

Campese VD, Kim IT, Rojas G, LeDoux JE (2017) Pavlovian extinction and recovery effects in aversive Pavlovian to instrumental transfer. Front Behav Neurosci 11:179.

Chen CF, Barnes DC, Wilson DA (2011) Generalized vs. stimulus-specific learned fear differentially modifies stimulus encoding in primary sensory cortex of awake rats. J Neurophysiol 106:3136-3144. 
Corbit LH, Balleine BW (2005) Double dissociation of basolateral and central amygdala lesions on the general and outcome-specific forms of pavlovian-instrumental transfer. J Neurosci 25:962-970.

Corbit LH, Janak PH, Balleine BW (2007) General and outcome-specific forms of Pavlovian-instrumental transfer: the effect of shifts in motivational state and inactivation of the ventral tegmental area. Eur J Neurosci 26:3141-3149.

Coutureau E, Killcross S (2003) Inactivation of the infralimbic prefrontal cortex reinstates goal-directed responding in overtrained rats. Behav Brain Res 146:167-174.

Delamater AR (1996) Effects of several extinction treatments upon the integrity of Pavlovian stimulus-outcome associations. Anim Learn Behav 24:437-449.

Delamater AR (2012) On the nature of CS and US representations in Pavlovian learning. Learn Behav 40:1-23.

Deslauriers J, Toth M, Der-Avakian A, Risbrough VB (2018) Current status of animal models of posttraumatic stress disorder-behavioral and biological phenotypes, and future challenges in improving translation. Biol Psychiatry 83:895-907.

Díaz-Mataix L, Debiec J, LeDoux JE, Doyère V (2011) Sensory-specific associations stored in the lateral amygdala allow for selective alteration of fear memories. J Neurosci 31:9538-9543.

Flaherty CF, Largen J (1975) Within-subjects positive and negative contrast effects in rats. J Comp Physiol Psychol 88:653-664.

Flaherty CF, Coppotelli C, Grigson PS, Mitchell C, Flaherty JE (1995) Investigation of the devaluation interpretation of anticipatory negative contrast. J Exp Psychol Anim Behav Process 21:229-247.

Ghosh S, Chattarji S (2015) Neuronal encoding of the switch from specific to generalized fear. Nat Neurosci 18:112-120.

Herry C, Johansen JP. Nat Neurosci (2014) Encoding of fear learning and memory in distributed neuronal circuits. Nat Neurosci 17:1644-1654.

Izquierdo A, Suda RK, Murray EA (2004) Bilateral orbital prefrontal cortex lesions in rhesus monkeys disrupt choices guided by both reward value and reward contingency. J Neurosci 24:7540-7548.

Janak PH, Tye KM (2015) From circuits to behaviour in the amygdala. Nature 517:284-292.

Johnson LR, Hou M, Ponce-Alvarez A, Gribelyuk LM, Alphs HH, Albert L, Brown BL, Ledoux JE, Doyère V (2008) A recurrent network in the lateral amygdala: a mechanism for coincidence detection. Front Neural Circuits 2:3.

Kattner F, Green CS (2016) Transfer of dimensional associability in human contingency learning. J Exp Psychol Anim Learn Cogn 42:15-31.

Krabbe S, Gründemann J, Lüthi A (2018) Amygdala inhibitory circuits regulate associative fear conditioning. Biol Psychiatry 83:800-809.

LeDoux JE, Pine DS (2016) Using neuroscience to help understand fear and anxiety: a two-system framework. Am J Psychiatry 173:1083-1093.
LeDoux JE, Moscarello J, Sears R, Campese V (2017) The birth, death and resurrection of avoidance: a reconceptualization of a troubled paradigm. Mol Psychiatry 22:24-36.

Le Pelley ME, Beesley T, Griffiths O (2014) Relative salience versus relative validity: cue salience influences blocking in human associative learning. J Exp Psychol Anim Learn Cogn 40:116-132.

Marchant NJ, Whitaker LR, Bossert JM, Harvey BK, Hope BT, Kaganovsky K, Adhikary S, Prisinzano TE, Vardy E, Roth BL, Shaham Y (2016) Behavioral and physiological effects of a novel kappa-opioid receptor-based DREADD in rats. Neuropsychopharmacology 41:402-409.

Paxinos G, Watson C (2013) The Rat Brain in Stereotaxic Coordinates. 7th Edition. London: Academic Press.

Pickens CL, Saddoris MP, Gallagher M, Holland PC (2005) Orbitofrontal lesions impair use of cue-outcome associations in a devaluation task. Behav Neurosci 119:317-322.

Rabinak CA, Orsini CA, Zimmerman JM, Maren S (2009) The amygdala is not necessary for unconditioned stimulus inflation after Pavlovian fear conditioning in rats. Learn Mem 16:645-654.

Rescorla RA (1973) Effect of US habituation following conditioning. J Comp Physiol Psychol 82:137-143.

Rescorla RA (1974) Effect of inflation of the unconditioned stimulus value following conditioning. J Comp Physiol Psychol 86:101-106.

Rescorla RA (1991) Associations of multiple outcomes with an instrumental response. J Exp Psychol Anim Behav Process 17:465-474.

Rescorla RA (1996) Preservation of Pavlovian associations through extinction. Q J Exp Psychol 49:245-258.

Schoenbaum G, Setlow B, Nugent SL, Saddoris MP, Gallagher M (2003a) Lesions of orbitofrontal cortex and basolateral amygdala complex disrupt acquisition of odor-guided discriminations and reversals. Learn Mem 10:129-140.

Schoenbaum G, Setlow B, Saddoris MP, Gallagher M (2003b) Encoding predicted outcome and acquired value in orbitofrontal cortex during cue sampling depends upon input from basolateral amygdala. Neuron 39:855-867.

Sengupta A, Yau JOY, Jean-Richard-Dit-Bressel P, Liu Y, Millan EZ, Power JM, McNally GP (2018) Basolateral amygdala neurons maintain aversive emotional salience. J Neurosci 38:3001-3012.

Staples-Bradley LK, Treanor M, Craske MG (2018) Discrimination between safe and unsafe stimuli mediates the relationship between trait anxiety and return of fear. Cogn Emot 32:167-173.

Vardy E, Robinson JE, Li C, Olsen RHJ, DiBerto JF, Giguere PM, Sassano FM, Huang XP, Zhu H, Urban DJ, White KL, Rittiner JE, Crowley NA, Pleil KE, Mazzone CM, Mosier PD, Song J, Kash TL, Malanga CJ, Krashes MJ, et al. (2015) A new DREADD facilitates the multiplexed chemogenetic interrogation of behavior. Neuron 86:936-946. 